\title{
Percepción comunitaria y procesos de Gestión Integrada de Zonas Costeras en el Pacífico Norte de Costa Rica
}

\author{
Astrid Sánchez-Jiménez ${ }^{1}$, Álvaro Morales-Ramírez ${ }^{2,3}$, Jimena Samper-Villarreal², \\ Celeste Sánchez-Noguera ${ }^{1,2}$ \\ 1. Programa de Posgrado en Gestión Integrada de Áreas Costeras Tropicales (GIACT), Universidad de Costa Rica, \\ astrid.sanchez@ucr.ac.cr \\ 2. Centro de Investigación en Ciencias del Mar y Limnología (CIMAR), Ciudad de la Investigación, Universidad de \\ Costa Rica, San Pedro, 11501-2060 San José, Costa Rica, alvaro.morales@ucr.ac.cr \\ 3. Escuela de Biología, Universidad de Costa Rica, San Pedro, 11501-2060 San José, Costa Rica
}

\author{
Recibido 09-X-2013. Corregido 22-V-2014. Aceptado 27-IX-2014.
}

\begin{abstract}
Community Perception and processes of Integrated Coastal Zone Management in the North Pacific of Costa Rica. The tendencies in perception on use and management of marine and coastal resources in the North Pacific of Costa Rica were studied through consultation workshops in the communities of Cuajiniquil, Villarreal, Montezuma, and surrounding areas. A total of 112 people from eight communities attended the workshops. Overall, there was higher attendance by men than by women. The main marine resources identified were fisheries and beach/waves; highlighted activities were fishing and tourism. The Costa Rican Institute of Fishing and Aquaculture (Incopesca) was identified as having deficient participation in the management of resources, besides was assigned low participation to the artisanal fishing and tourism. The involvement of women as leaders in Montezuma was highlighted, which emphasized the need to promote the autonomy of coastal women so that more female citizens are in the condition to contribute as legitimate stakeholders in the decision making process. The main challenges detected were illegal fishing in Marine Protected Areas and overfishing. As solutions, the communities proposed elements of sustainable fishing, and the elimination of trawling and compressor diving. The consultation process revealed the need to propose economic alternatives to fishing, the education of local communities, focus more attention on management categories that do not prohibit artisanal fishing, and to promote the participation of the civil society in the decision making process. Existing mechanisms should be used to promote community inclusion and participatory processes. Rev. Biol. Trop. 62 (Suppl. 4): 139-149. Epub 2014 Diciembre 01.
\end{abstract}

Key words: integrated coastal zone management (ICZM), community perception, marine resources, participatory processes, sustainable fishing.

Las zonas costeras representan áreas de gran importancia mundial en términos económicos, de biodiversidad y demografía, que se caracterizan por albergar recursos que son aprovechados por distintos sectores (Worm et al., 2006). El rápido crecimiento de las poblaciones litorales, la pobreza y la sobreexplotación de los recursos marinos y pesqueros, han contribuido con la degradación ambiental de muchos sitios. En las áreas costeras tropicales la pérdida de hábitats críticos, la sobreexplotación y la reducción de la biodiversidad, han sido señaladas como unas de las principales amenazas (Cicin-Sain \& Knecht, 1998; Westmacott, 2002).

La Gestión Integrada de la Zonas Costeras (GIZC) se ha propuesto como una de las herramientas principales para enfrentar la presión sobre las costas en Latinoamérica (Lemary, Vaughan, Rodríguez, Christy, Agüero \& Rodríguez, 1997; Yañez-Arancibia, 1999; Barragán, 2012), debido a que su finalidad es 
el aprovechamiento sostenible de los ambientes y recursos costeros tomando como referencia el contexto ambiental, político y socioeconómico de cada región (Barragán, 2003). Las costas de Costa Rica están expuestas a múltiples amenazas que han requerido de un abordaje integral de los retos existentes (Morales-Ramírez, Silva-Benavides \& González-Gairaud, 2010; Morales-Ramírez, 2013).

Las comunidades costeras poseen conocimientos sobre el uso histórico y actual de los recursos. Se ha demostrado que utilizar esa información en conjunto con datos de carácter biológico permite analizar integralmente una problemática y mejorar la legitimidad de gobernanza de las costas (Castilla, Gelcich \& Defeo, 2007; Marshall, Marshall, Abdulla \& Rouphael, 2010; Correa, Turbay \& Vélez, 2011). Algunos trabajos de percepción ciudadana con respecto al tema medioambiental han sido publicados en los últimos años en el país (Pernudi, Solórzano, Sandoval \& Vargas, 2004, 2005; Centeno, González \& López, 2012), pero pocos se han enfocado en las comunidades costeras (Castellón-Zelaya, 2006; Pereira \& Sierra, 2009).

El presente estudio tiene como objetivo ampliar el nivel de conocimiento en relación al estado, uso y manejo de los recursos marinocosteros del Pacífico Norte de Costa Rica, analizando las tendencias en percepción comunitaria por medio de un proceso participativo de consulta.

\section{MATERIALES Y MÉTODOS}

Sitio de estudio: Se seleccionaron tres puntos focales de trabajo a lo largo del Pacífico Norte: 1) Cuajiniquil (región Norte, $10^{\circ} 56^{\prime} 36.08^{\prime \prime} \mathrm{N}, 85^{\circ} 41^{\prime} 09.10^{\prime \prime} \mathrm{O}$ ); 2) Villarreal (región intermedia, $10^{\circ} 18^{\prime} 26.12^{\prime \prime} \mathrm{N}$, 8548'22.80"O); y 3) Montezuma (región Sur, 9`39'24.41'”N, 8504'00.67'O). Dadas las posibilidades logísticas, la distribución geográfica de estos tres puntos focales se consideró eficiente para representar las comunidades ubicadas a lo largo de la costa del Pacífico Norte.
Recolección de la información: Para conocer la percepción local sobre uso y manejo de los recursos marino-costeros del Pacífico Norte de Costa Rica, se llevó a cabo un taller de consulta en cada punto focal mencionado anteriormente (tres talleres en total), durante los meses de marzo, mayo y agosto del 2012.

- Convocatoria a los talleres: Se colocaron afiches y entregaron invitaciones de previo a cada taller. Los grupos de interés en cada comunidad lo conformaron: líderes y lideresas de la comunidad, habitantes locales, agrupaciones religiosas, sector empresarial de la zona, representantes del sector pesca y turismo, representantes del Sistema Nacional de Áreas de Conservación (SINAC), instituciones estatales y organizaciones no gubernamentales (ONG). Fueron invitadas las comunidades aledañas al sitio de ejecución de cada taller.

- Realización de los talleres: Se utilizaron las instalaciones más viables en cuanto a facilidades y acceso para los participantes, tales como salones comunales y restaurantes. Los talleres se basaron en metodologías probadas para procesos de consulta (Bunce, Townsley, Pomeroy \& Pollnac, 2000; Bunce \& Pomeroy, 2003), aplicadas por un equipo de facilitadores que fomentó la intervención equitativa de los participantes y que registró los resultados a través de fotografías, notas y recolección del material utilizado.

\section{- Áreas temáticas abordadas:}

\section{Identificación y aprovechamiento de los recursos marino-costeros}

Se utilizó el concepto de recurso marino descrito en la ley orgánica del ambiente (Ley No7554, 1995). Fueron delimitadas las áreas de interés en cada comunidad, se listaron los recursos marino-costeros presentes y las principales formas de aprovechamiento. 


\section{Manejo costero}

Se explicaron algunos términos a los participantes. Manejo costero (uso sostenible a largo plazo de los recursos costeros y mantenimiento de sus beneficios) (Clark, 1995). Actor (persona física o jurídica involucrada como usuario o administrador de los recursos marinos) según DNP, (2008). Para efectos de los talleres se entendió participación como las acciones relacionadas con la administración de los recursos, y dependencia como el beneficio directo o indirecto derivado del uso de los recursos marinos. Una vez explicados los conceptos, los asistentes reconocieron los actores y su grado de dependencia/participación en el manejo. Fueron enumerados los retos y dificultades para el manejo y se identificaron propuestas para el manejo de los recursos marino-costeros.

\section{Análisis de la información obtenida:} Para analizar la percepción, se listaron las respuestas de los asistentes a los talleres en relación a las áreas temáticas de interés. Fue utilizada la metodología empleada para codificar preguntas abiertas de entrevistas (Sampieri, Collado, Lucio, \& Pérez, 1998), en la cual se da un nombre genérico a respuestas similares para facilitar la agrupación de los resultados y realizar así un análisis de la frecuencia de mención. En este caso, la frecuencia con que una respuesta se mencionó en el total de talleres de consulta.

\section{RESULTADOS}

Caracterización de la población participante en los talleres: En total se registraron 112 participantes en los talleres, provenientes de ocho comunidades: $62 \%$ procedente de la región Norte (Cuajiniquil y Cangrejal), 34\% de la región Sur (Montezuma, Cabuya, Pochote, Tambor y Cabo Blanco) y 4\% de la región Central (Villarreal de Tamarindo) (Fig.1.A). Hubo un mayor número de participantes hombres $(66 \%)$ que de mujeres (34\%), en contraste con el último censo poblacional que registró más mujeres en los cantones de Santa Cruz y Puntarenas, donde se ubican las comunidades consultadas (INEC, 2011) (Fig. 1.B).

Los sectores representados en los talleres fueron: habitantes locales, grupos comunitarios organizados, organizaciones no gubernamentales (ONG), sector económico (turismo, pesca y comercio) y funcionarios estatales (SINAC, Fuerza Pública, Municipalidad) En Villarreal únicamente se contó con la participación de representantes del sector de pesca deportiva. Asistieron representantes del SINAC: Reserva Absoluta Cabo Blanco (RNACB) y Parque Nacional Santa Rosa (PNSR) (Figs. 2.A y 2.B).

Delimitación de las áreas de interés: $\mathrm{El}$ área de interés para cada taller fue demarcada con base en las áreas marino-costeras en que se realizan actividades económicas según lo indicaron los participantes: pesca artesanal, turismo, otras (proyectos de conservación); y de acuerdo a la presencia de ambientes marinos y especies de importancia (cetáceos, tortugas y manglar) (Fig. 3). En el taller realizado en Cuajiniquil se señalaron de manera exclusiva lugares de importancia pesquera, en el de Villarreal y Montezuma principalmente áreas de relevancia turística y en menor medida de importancia pesquera.

Identificación y aprovechamiento de los recursos marino-costeros: En todos los talleres se destacó la importancia del recurso pesquero y del recurso playa/olas (Cuadro 1). Asociados a estos dos recursos, las actividades pesqueras y el turismo fueron señalados como las principales formas de aprovechamiento. Las tortugas marinas y los recursos humano e hídrico fueron destacados en Montezuma (Cuadro 1), zona en la que se identificaron dos servicios ambientales relacionados al recurso playa, la belleza escénica y la conservación.

\section{Manejo costero}

Reconocimiento $\mathbf{y}$ análisis de actores: De acuerdo con los conceptos previamente 

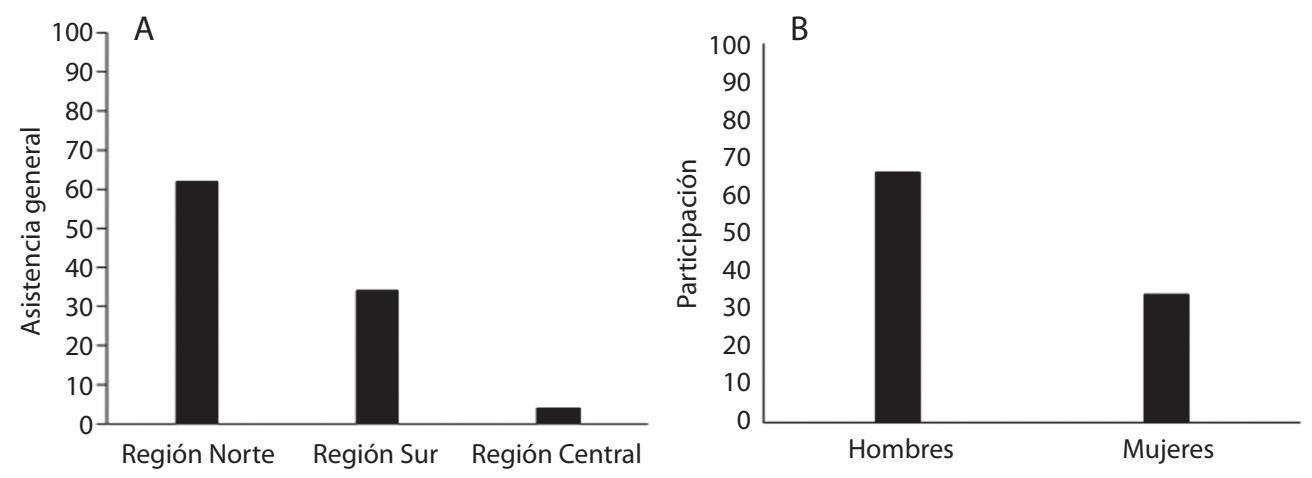

Fig. 1. (A) Asistencia total (\%) a los talleres según región de origen en el Pacífico Norte y (B) Asistencia total (\%) a los talleres de mujeres y hombres.
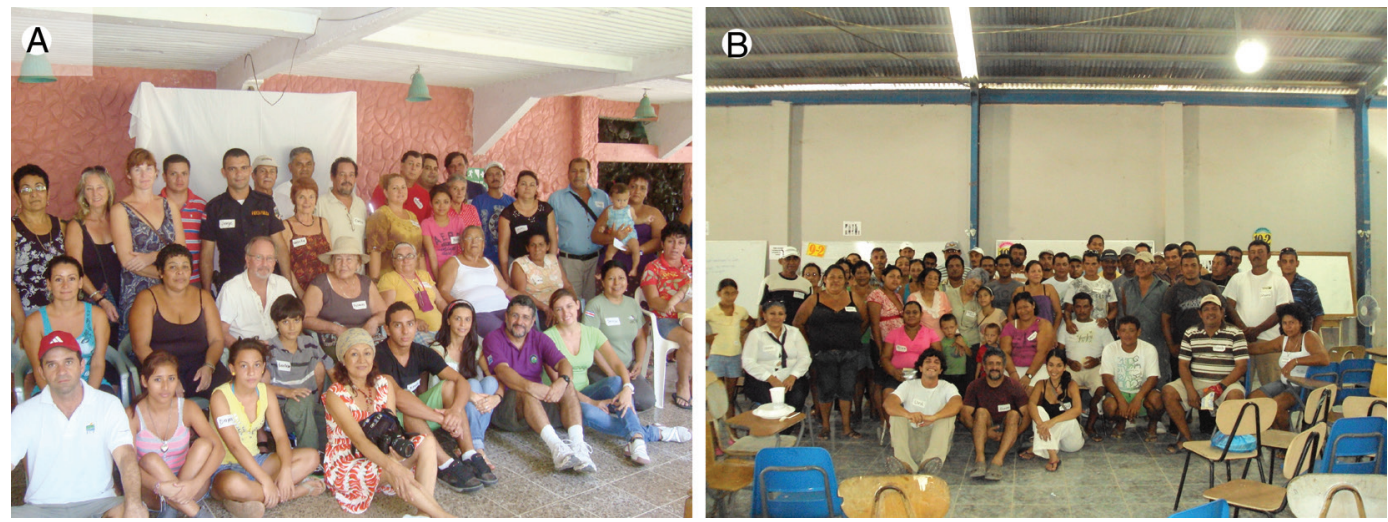

Fig. 2. (A) Personas asistentes al taller realizado en Montezuma, agosto de 2012. (B) Personas asistentes al taller realizado en Cuajiniquil, marzo de 2012.

definidos (ver sección de materiales y métodos), el proceso de consulta permitió reconocer 21 actores en la región, con distintos grados de participación y dependencia (Cuadro 2). A nivel general los asistentes a los talleres opinaron que los actores con una dependencia más alta (es decir aquellos que más se benefician de los recursos marino-costeros), son los del sector de pesca artesanal y deportiva, seguidos por los del sector turismo. En lo que respecta a la participación en la administración de los recursos marino-costeros, el sector de pesca artesanal fue identificado con una baja participación y el de turismo se percibió con una participación baja para la mayoría de las personas consultadas, excepto en Montezuma.
A varias instituciones gubernamentales se les asignó una baja participación, especialmente a Incopesca, institución que además fue señalada como poco eficiente para administrar los recursos marinos. Se destacó la intervención en la administración de los recursos marinos, del sector de pesca deportiva en Villarreal, lideresas comunales y de algunos grupos comunitarios organizados de Montezuma (Cuadro 2).

Caracterización de retos para el manejo: En un $100 \%$ de los talleres de consulta se identificaron como retos, el uso de artes de pesca no selectivas, refiriéndose a técnicas que pescan especies no objetivo y que impiden que 

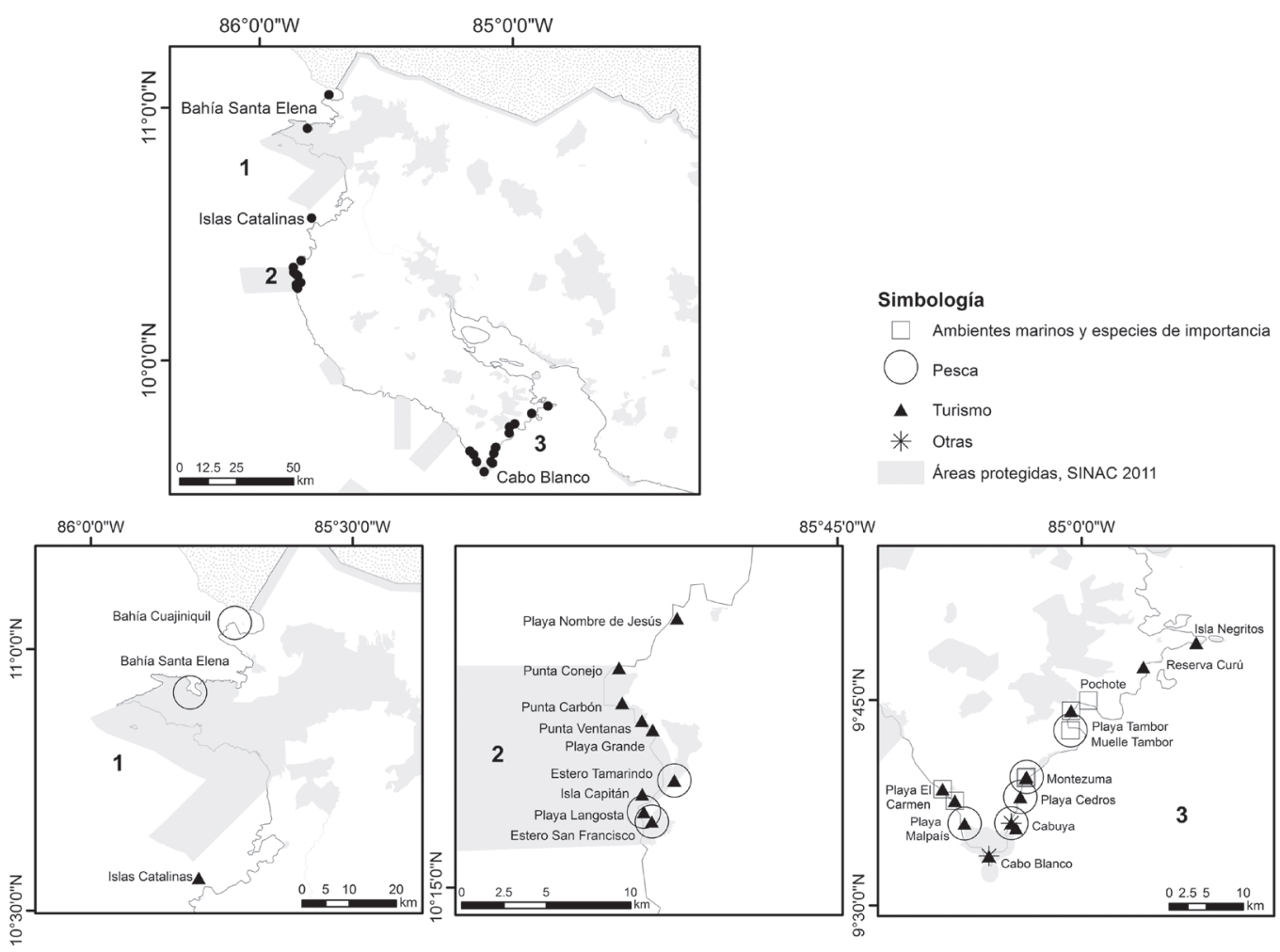

Fig. 3. Delimitación de las áreas de interés en los tres talleres de consulta realizados en el Pacífico Norte. 1=Cuajiniquil, 2=Villarreal, 3=Montezuma. Mapa: Catalina Benavides, SIGMAR, CIMAR, 2013.

\section{CUADRO 1}

Recursos marino-costeros identificados en los diferentes talleres de consulta y frecuencia de talleres en que fueron mencionados. Se detallan nombres científicos de los recursos pesqueros de importancia comercial

\begin{tabular}{llcc}
\multicolumn{1}{c}{ Nombre común } & \multicolumn{1}{c}{ Nombre científico } & Frecuencia de mención (\%) & Taller \\
Playa/olas & - & 100 & $\mathrm{C}, \mathrm{V}, \mathrm{M}$ \\
Recurso pesquero & - & 100 & $\mathrm{C}, \mathrm{V}, \mathrm{M}$ \\
Langosta espinosa verde & Panulirus gracilis Streets, 1871 & 100 & $\mathrm{C}, \mathrm{V}, \mathrm{M}$ \\
Pez marlín rayado & Kajikia audax (Philippi, 1887) & 67 & $\mathrm{C}, \mathrm{V}$ \\
Pez vela & Istiophorus platypterus (Shaw \& Nodder 1792) & 67 & $\mathrm{C}, \mathrm{V}$ \\
Pez dorado & Coryphaena hippurus Linnaeus, 1758 & 67 & $\mathrm{C}, \mathrm{V}$ \\
Atún aleta amarilla & Thunnus albacares (Bonnaterre, 1788) & 67 & $\mathrm{C}, \mathrm{V}$ \\
Pepino de mar & Isostichopus fuscus (Ludwig, 1875) & 67 & $\mathrm{C}, \mathrm{V}$ \\
Pulpo & Octopus vulgaris (Lamarck, 1798) & 67 & $\mathrm{C}, \mathrm{M}$ \\
Pez gallo & Nematistius pectoralis (Gill, 1862) & 33 & $\mathrm{~V}$ \\
Cambute & Strombus galeatus (Swainson, 1823) & 33 & $\mathrm{M}$ \\
Piangüa, ostra, almeja & Varias especies & 33 & $\mathrm{C}, \mathrm{M}, \mathrm{V}$ \\
Camarón & Varias especies & 33 & $\mathrm{M}$ \\
Tortugas marinas & & 33 & $\mathrm{M}$ \\
Recurso humano & - & 33 & $\mathrm{M}$ \\
Recurso hídrico & - & 33 & $\mathrm{M}$
\end{tabular}

Taller realizado en tres comunidades, $\mathrm{C}=$ Cuajiniquil, $\mathrm{V}=$ Villarreal, $\mathrm{M}=$ Montezuma. 
CUADRO 2

Actores identificados y el grado de dependencia y participación la administración de los recursos marino costeros, junto con la frecuencia de talleres en que fueron reconocidos como actores.

\begin{tabular}{|c|c|c|c|}
\hline Actores & Participación & Dependencia & Frecuencia mención(\%) \\
\hline Pesca deportiva & Alta $^{\mathrm{C} \mathrm{V}}$ & Alta $^{\mathrm{C}} \mathrm{V}$ & 67 \\
\hline Comité del Agua Cabo Blanco & Alta $^{\mathrm{M}}$ & Alta $^{\mathrm{M}}$ & 33 \\
\hline Municipalidad & Alta $^{\mathrm{M}}$ & Alta $^{\mathrm{M}}$, Intermedia ${ }^{\mathrm{C}}$ & 67 \\
\hline Asoc. de Desarrollo Cabo Blanco & Alta $^{\mathrm{M}}$ & Intermedia $^{\mathrm{M}}$ & 33 \\
\hline Iglesias & Alta $^{\mathrm{M}}$ & Intermedia $^{\mathrm{M}}$ & 33 \\
\hline Líderes y lideresas comunales & Alta $^{\mathrm{M}}$ & Intermedia $^{\mathrm{M}}$ & 33 \\
\hline Sector Turismo & $\mathrm{Alta}^{\mathrm{M}}, \mathrm{Baja}^{\mathrm{C}}, \mathrm{Nula}^{\mathrm{V}}$ & Alta $^{\mathrm{C}} \mathrm{V}$, Intermedia $^{\mathrm{M}}$ & 100 \\
\hline ASADAS & Intermedia $^{\mathrm{M}}$ & Alta $^{\mathrm{M}}$ & 33 \\
\hline ONG (ASVO) & Intermedia $^{\mathrm{M}}$ & Alta $^{\mathrm{M}}$ & 33 \\
\hline Personas locales & Intermedia $^{\mathrm{M}}$ & Alta $^{\mathrm{M}}$ & 33 \\
\hline UCR/UNA & Intermedia $^{\mathrm{M}}$ & Alta $^{\mathrm{M}}$ & 33 \\
\hline MINAE/SINAC & Intermedia $^{\mathrm{C}}, \mathrm{Baja}^{\mathrm{M}}, \mathrm{Nula}^{\mathrm{V}}$ & $\mathrm{Alta}^{\mathrm{M}}$, Intermedia $^{\mathrm{C}} \mathrm{V}$ & 100 \\
\hline SNG & Intermedia $^{\mathrm{C}}, \mathrm{Baja}^{\mathrm{M}}$ & Intermedia $^{\mathrm{M}}, \mathrm{Baja}^{\mathrm{C}}$ & 67 \\
\hline Asoc. de pescadores Montezuma & Baja $^{\mathrm{M}}$ & Intermedia $^{\mathrm{M}}$ & 33 \\
\hline Fuerza Pública & Baja $^{\mathrm{M}}$ & Intermedia $^{\mathrm{M}}$ & 33 \\
\hline MAG & Baja $^{\mathrm{M}}$ & Intermedia $^{\mathrm{M}}$ & 33 \\
\hline Asociación Desarrollo Tambor & Baja $^{\mathrm{M}}$ & Baja $^{\mathrm{M}}$ & 33 \\
\hline AyA & Baja $^{\mathrm{M}}$ & Baja $^{\mathrm{M}}$ & 33 \\
\hline ONG (Marviva) & Baja $^{\mathrm{C}}$ & Baja $^{\mathrm{C}}$ & 33 \\
\hline Pesca artesanal & Baja $^{\mathrm{C}}$, NulaV & Alta ${ }^{\mathrm{C}} \mathrm{V}$ & 67 \\
\hline Incopesca & Baja $^{\mathrm{C} M} \mathrm{Nula}^{\mathrm{V}}$ & $\mathrm{Baja}^{\mathrm{C} V}, \mathrm{Alta}^{\mathrm{M}}$, & 100 \\
\hline
\end{tabular}

Taller realizado en tres comunidades, Cuajiniquil $=\mathbf{C}$, Villarreal $=\mathbf{V}$ y Montezuma $=\mathbf{M}$.

los organismos juveniles se escapen, como la pesca de camarón por arrastre. Así mismo, se señalaron los temas de la pesca ilegal dentro de AMPs, la extracción de langosta sin control, la sobreexplotación del recurso pesquero, la baja participación de las entidades estatales en la administración de los recursos marinos y la escasa participación formal de las comunidades en la toma de decisiones de dichos recursos.

Entre otros problemas importantes pero menos frecuentes (identificados en un 33\% de los talleres), se mencionan la contaminación de las aguas, la gestión de los residuos sólidos y amenazas para las tortugas marinas como la contaminación lumínica y la compactación de arena por vehículos en la playa.

Propuestas para el manejo de los recursos: En cada taller se desarrollaron planteamientos para el manejo de los recursos marino-costeros, los cuales tienen en común dos ejes temáticos: 1) Desarrollo de proyectos de pesca sostenible que incluye varios aspectos: pesca de individuos con tallas mínimas, identificación de áreas de crianza de peces, la no pesca en arrecifes, establecimiento de vedas para facilitar la recuperación de especies, prohibición de la pesca de camarón por arrastre y de buceo con compresor. 2) Proceso educativo y de capacitación política para promover la participación ciudadana en la toma de las decisiones. Al respecto, algunas personas proponen la política como un mecanismo para incidir adecuadamente en los asuntos públicos de interés.

En dos de los talleres realizados, fue sugerida la posibilidad de pescar artesanalmente dentro de AMPs. Entre otras recomendaciones se incluyen: una participación adecuada 
en la administración de los recursos marinos por parte de las instituciones gubernamentales (principalmente de las universidades públicas e Incopesca), la necesidad de señalar los límites marinos de las áreas protegidas con boyas para identificar los sitios con restricciones de pesca, una estandarización de las licencias pesqueras para que una misma licencia autorice varios permisos de uso. Vigilar más efectivamente el recurso tortugas marinas, la posibilidad de establecer denuncias de carácter anónimo en instituciones estatales y que la municipalidad asuma la recolección de basura de la zona.

\section{DISCUSIÓN}

La información generada por medio de talleres de consulta permitió ampliar el conocimiento, en relación a las percepciones de actores locales, sobre el estado de conservación, uso y manejo de los recursos marino-costeros en el Pacífico Norte de Costa Rica. Se aprecia que las personas consultadas consideran importante para el manejo de los recursos de la región, el abordaje de dos áreas principales: 1) Transición hacia una pesca sostenible y el desafío de la pesca ilegal dentro de AMPs, 2) Mayor participación ciudadana en las decisiones de manejo de los recursos marinos.

\section{1) Transición hacia una pesca sostenible} y el desafío de la pesca dentro de AMPs: $\mathrm{La}$ sobreexplotación del recurso pesquero, percepción que se resaltó en la consulta, ha sido ampliamente registrada en las costas tropicales del mundo (Westmacott, 2002). La eliminación de la pesca de arrastre fue sugerida como medida básica para abordar el tema de la sobrepesca. Mientras se trabajaba en la presente investigación, la Sala IV, basada en el interés público del país, declaró inconstitucional el arte de pesca de camarón por arrastre, lo cual implica que Incopesca no podrá otorgar ningún permiso, ni tampoco renovar los vencidos ni reactivar los inactivos (Poder Judicial, 2013). Esta decisión podría contribuir a recuperar algunas de las especies de peces y otros grupos que se pescan incidentalmente en grandes cantidades con la técnica de arrastre de camarón (Arana, Wehrtmann, Orellana, Nielsen-Muñoz \& VillalobosRojas, 2013).

Los actores consultados identificaron la langosta como uno de los principales recursos marino-costeros de la región, pero a su vez propusieron eliminar su captura mediante el buceo con compresor. Dicho arte de pesca no puede ser clasificado como sostenible medioambientalmente, ya que su eficiencia está condicionada a la destreza y subjetividad del pescador (Naranjo 2010). Este aspecto plantea la necesidad de implementar otros artes de pesca, como podrían serlo las nasas. Naranjo, (2010) recomienda evaluar la viabilidad técnica de este tipo de iniciativas y no perder de perspectiva que los pescadores requerirían de un acompañamiento para hacer la transición a este nuevo arte, incluyendo una inversión económica inicial.

Sugerencias planteadas en el proceso de consulta como el uso de artes de pesca más selectivas, la pesca de individuos que han alcanzado la madurez sexual y la identificación de áreas de crianza de peces, podrían considerarse indicadores de la disposición para desarrollar proyectos de pesca sostenible en el Pacífico Norte del país. Se recomienda identificar con claridad los requerimientos para el desarrollo de una pesca sostenible, ya que sería erróneo asumir que toda iniciativa comunitaria de manejo pesquero, cumpliría por defecto con los principios y criterios técnicos de la sostenibilidad medioambiental y social establecidos internacionalmente (ver MSC, 2012). En efecto, no existe aún en el país ninguna pesquería certificada como sostenible de manera oficial, aunque cabe destacar que actualmente hay pescadores artesanales dirigiendo esfuerzos en ese sentido (Soto, 2014).

Las estadísticas de pesca para la región de Guanacaste muestran una disminución a partir del 2001, con un nivel mínimo de captura en el 2007 (INCOPESCA, 2013), lo cual podría estar influyendo en la incursión de pescadores artesanales en áreas donde la pesca no es permitida. Sin embargo, la propuesta de algunos habitantes consultados de legalizar la pesca 
artesanal en AMPs, no precisamente contribuiría a mejorar su situación socioeconómica, en especial si se considera la relación encontrada entre la presencia de áreas de no pesca y el aumento significativo de la productividad pesquera en los alrededores, tras analizar múltiples experiencias locales de conservación (Worm et al., 2006).

Marshall et al., (2010), han demostrado que la disposición para apoyar iniciativas de conservación tiende a incrementarse cuando hay múltiples fuentes de ingresos, por lo que introducir alternativas económicas en zonas altamente dependientes de la pesca podría ayudar a diversificar la economía, reducir la dependencia sobre el recurso pesquero y propiciar un ambiente favorable para el desarrollo de proyectos de conservación marina (Rowe, 2011). El turismo podría ser una de esas alternativas, siempre y cuando el modelo a implementar persiga el interés público del país (Honey, Vargas \& Durham, 2010).

La educación y el involucramiento de las comunidades, son herramientas importantes para abordar el reto de la pesca ilegal (Cobas, 2007; Rowe, 2011). Es necesario demostrar apropiadamente a los pobladores que la protección de la biodiversidad marina y el mantenimiento de los bienes/servicios están relacionados (Worm et al., 2006). Medidas como el apoyo a la gestión de las AMPs que ya están establecidas en el Pacífico Norte, en conjunto con el desarrollo de proyectos de pesca sostenible en los alrededores, podrían contribuir con el aumento de la productividad pesquera de la región.

El proceso de consulta reveló que es prioritario trabajar en: a) evaluar alternativas económicas a la pesca, b) la educación de los usuarios de los recursos marinos, e c) implementar proyectos de pesca sostenible en los alrededores de las AMPs ya establecidas. En caso de crear nuevas AMPs en el Pacífico Norte, se recomienda enfocar la atención en categorías de manejo como las Reservas Marinas y Áreas Marinas de Manejo, que no prohíben la pesca artesanal (Alvarado, Herrera, Corrales, Asch \& Paaby, 2011), pero que son adecuadas para implementarse en zonas con potencial para actividades pesqueras sostenibles (Cajiao, Rodríguez \& Lobo, 2010).

2) Mayor participación ciudadana en las decisiones de manejo de los recursos marinos: Una participación poco eficiente de Incopesca en la administración de los recursos fue percibida en la consulta, lo cual podría estar asociado a la baja representatividad del sector de pesca artesanal y de otros actores en la toma de decisiones pesqueras asumidas por la institución (Quesada 2006). Los poblados costeros, para intentar canalizar sus propuestas, se han organizado en asociaciones/cooperativas o han establecido alianzas con $\mathrm{ONG}$ e instituciones gubernamentales, pero poco han intervenido en la toma de decisiones formales sobre el manejo de los recursos marinos (Solís-Rivera et al., 2012).

En los talleres de consulta, a pesar de haberse señalado cuán importantes son los grupos comunitarios organizados ya existentes, los asistentes coinciden en que aún se requiere de otros espacios para opinar e incidir en los temas de interés; y sugieren que estos deberían venir acompañados de un proceso educativo que promueva no sólo un mayor aporte de los pobladores, sino uno del tipo asertivo. Si se considera por ejemplo, la baja participación de las mujeres en los talleres de consulta pero a su vez la injerencia de lideresas comunales en la gestión de los recursos marinos de Montezuma, surge la necesidad de trabajar en la construcción de la autonomía y poderío de la mujer (Lagarde, 2000), de manera tal que más ciudadanas puedan contribuir como un actor legítimo en procesos de manejo costero de la región; lo cual es aplicable también para otros sectores clave de la población.

Experiencias en la gestión de pesquerías bentónicas en América Latina (Castilla \& Defeo, 2001), demuestran que procesos participativos como el comanejo, han empezado a retribuir económicamente a pobladores locales en Chile, a raíz de una mejora en los indicadores bio-económicos de la pesca (e.g. captura por unidad de esfuerzo, etc.) (Castilla et al., 
2007); para lograr esto, fue necesario en una primera etapa ampliar la información científica disponible, incluyendo aquella brindada por los pescadores y legitimar su participación en el proceso de gestión (Castilla \& Defeo, 2001). En Costa Rica, existen comunidades que se han involucrado en iniciativas como el comanejo de aspectos financieros en algunos parques nacionales (CCAD/PNUD/GEF, 2003), pero finalmente fueron detectados vacíos legales que imposibilitaron al Estado apoyar adecuadamente este tipo de procesos (CGR, 2005).

De llegar a existir un contexto jurídico en el país que respalde procesos de comanejo u otras herramientas para la gobernanza marina de carácter participativo, sería prioritario delimitar con claridad las unidades de gestión (Castilla \& Defeo, 2001). El grupo que va a participar en la acción de manejo debería ser representativo de los distintos intereses, con comunidades geográficas (e.g., Puerto Jiménez) más que con comunidades sectoriales (e.g., sector de pesca o turismo). Conjuntamente, iniciativas de este tipo deberían estar visualizadas dentro de un concepto amplio en el que se rinden cuentas y son asumidas responsabilidades que trascienden el aspecto monetario, además en el que la toma de decisiones persigue el interés público del país para enmarcar el alcance de proyectos de carácter local (M. Quesada-Alpízar, comunicación personal, 14 Agosto, 2013).

En la actualidad, en cuanto al manejo de los recursos marinos de la zona estudiada se sugiere aprovechar los órganos oficiales de participación del SINAC (Decreto Ejecutivo34433-08-MINAE), tal y como se ha venido haciendo, ya que estos representan vías existentes de inserción de la sociedad civil en la toma de decisiones; así mismo explorar sistemáticamente nuevas formas de incluir actores clave en estos procesos. En lo que respecta al ordenamiento de los recursos marinos, es fundamental el respaldo por medio de programas de monitoreo a largo plazo que brinden la información necesaria para adaptar constantemente las políticas de aprovechamiento de los recursos.
En futuros estudios relacionados con la percepción comunitaria en el Pacífico Norte del país, se recomienda desarrollar nuevos talleres de consulta $\mathrm{u}$ otras herramientas para la recolección de datos como las encuestas/ entrevistas, para lograr captar información de lugares no incluidos e impresiones de actores clave no consultados en esta oportunidad. La presente investigación muestra que la gobernanza marina de la región enfrenta retos, entre ellos la falta de una participación formal de las comunidades en el manejo de los recursos marino-costeros, pero a su vez cuenta con propuestas ciudadanas concretas con gran potencial para contribuir con una toma de decisiones más representativa de los distintos intereses de la región.

\section{AGRADECIMIENTOS}

Agradecemos sinceramente a las comunidades visitadas por su disposición a participar en el proceso, así como a Gleice Alves, Uirá Rocha y Angie Garita por su colaboración durante la realización de los talleres de consulta. Se agradece a Catalina Benavides y a Marco Quesada por sus aportes al manuscrito, a Victoria Quirós de Montezuma por las facilidades ofrecidas para la logística del taller en esta localidad. Apreciamos el apoyo de la Agencia Alemana de Cooperación (GiZ) y la Asociación Costa Rica por Siempre en el financiamiento del proyecto "Estudios Científicos en el Área Costera del Pacífico Norte". Esta es una contribución del proyecto 808-B2-400 adscrito a la Vicerrectoría de Investigación de la Universidad de Costa Rica coordinado por AMR.

\section{RESUMEN}

Las tendencias en la percepción sobre el uso y manejo de los recursos marino-costeros en el Pacífico Norte de Costa Rica se investigaron a través de talleres de consulta en las comunidades de Cuajiniquil, Villarreal, Montezuma y zonas aledañas. En total 112 personas provenientes de ocho comunidades asistieron a la convocatoria. En general se registró una mayor participación de hombres que de mujeres. Los principales recursos marinos identificados fueron los pesqueros y el de playa/olas; las actividades 
destacadas, la pesca y el turismo. Se asignó a Incopesca una deficiente participación en la administración de los recursos y se otorgó una baja participación a los sectores de pesca artesanal y turismo. Fue destacada la participación de lideresas en Montezuma, lo que planteó la necesidad de fomentar la autonomía de la mujer costeña para que más ciudadanas estén en condiciones de contribuir en la toma de decisiones como un actor legítimo. Los principales retos detectados fueron la pesca ilegal en las Áreas Marinas Protegidas y la sobreexplotación pesquera. Como soluciones, las comunidades propusieron elementos de pesca sostenible, además de la eliminación de la pesca de arrastre y de buceo con compresor. El proceso de consulta revela la necesidad de proponer alternativas económicas a la pesca, educar a las comunidades, enfocar la atención en categorías de manejo que no prohíban la pesca artesanal y promover la participación de la sociedad civil en la toma de decisiones. Se recomienda aprovechar los mecanismos ya existentes para la inclusión de la sociedad civil y de implementarse procesos participativos, que estos velen por el interés público del país aunque el proyecto sea de carácter local. Se evidencia que la gobernanza marina del Pacífico Norte del país enfrenta retos, entre ellos la falta de una gestión participativa de los recursos marino-costeros, pero a su vez cuenta con propuestas ciudadanas concretas que podrían contribuir con una toma de decisiones más representativa de los distintos intereses de la región.

Palabras clave: gestión integrada de las zonas costeras (GIZC), percepción comunitaria, recursos marinos, procesos participativos, pesca sostenible.

\section{REFERENCIAS}

Alvarado, J.J., Herrera, B., Corrales, L., Asch, J., \& Paaby, P. (2011). Identificación de las prioridades de conservación de la biodiversidad marina y costera en Costa Rica. Revista de Biología Tropical, 59: 829-842.

Arana, P.M., Wehrtmann, I.S., Orellana, J.C., NielsenMuñoz, V., \& Villalobos-Rojas, F. (2013). By-catch associated with fisheries of Heterocarpus vicarius (Costa Rica) and Heterocarpus reedi (Chile) (Decapoda: Pandalidae): a six-year study (2004-2009). Journal of Crustean Biology, 33: 198-209.

Barragán, J.M. 2003. La gestión costera en Latinoamérica: entre las experiencias nacionales y la necesidad de cooperación regional. Conferencia Invitada. X. Congreso Latinoamericano de Ciencias Marinas, San José, Costa Rica.

Barragán, J.M. (2012). Manejo Costero Integrado en Iberoamérica: Diagnóstico y propuestas para una nueva política pública (152 p). Cádiz, España: Red IBERMAR (CYTED).

Bunce, L., Townsley P., Pomeroy R., \& Pollnac, R. (2000). Socio-economic Manual for coral reef management.
Global Coral Reef Monitoring Network (251 p.). Townsville, Australia: Australian Institute of Marine Science.

Bunce, L. \& Pomeroy, R. (2003). Socioeconomic Monitoring Guidelines for Coastal Managers in the Caribbean. (SocMon Caribbean) (pp. 1-88). World Commission on Protected Areas and Australian Institute of Marine Science.

Cajiao, V., M. Rodríguez \& Lobo, A. (2010). Manual de legislación marino costera y pesquera de Costa Rica (1 ed.). San José, Costa Rica: Fundación Marviva.

Castellón-Zelaya, L. (2006). Percepción sobre el impacto de la actividad turística en una comunidad costera de Guanacaste. InterSedes: Revista de las Sedes Regionales, 7(13): 67-77.

Castilla, J. C., \& Defeo, O. (2001). Latin American benthic shellfisheries: emphasis on co-management and experimental practices. Reviews in Fish Biology and Fisheries, 11(1), 1-30.

Castilla, J. C., Gelcich, S., \& Defeo, O. (2007). Successes, lessons and projections from experience in marine benthic invertebrate artisanal fisheries in Chile. Fisheries management: progress towards sustainability, 23-42.

CCAD/PNUD/GEF. (2003). Proyecto para la consolidación del Corredor Biológico Mesoamericano. Estado actual del comanejo de áreas protegidas en Mesoamérica/Proyecto para la consolidación del Corredor Biológico Mesoamericano (1 ed.) Managua: Proyecto Corredor Biológico Mesoamericano.

Centeno, J., González, H. \& López, N. (2012). Percepción de las comunidades sobre el ambiente y la relación con los parques nacionales cercanos. En UNAIDESPO. (Ed.). Comunidades y Áreas Silvestres Protegidas: identidad, convivencia y conservación ambiental. (2 ed., pp. 51-209). Heredia, Costa Rica: Universidad Nacional. Instituto de Estudios Sociales en Población (IDESPO).

Cicin-Sain, B., \& Knecht, R. (1998). Integrated coastal and ocean management: concepts and practices (1 ed.). Washington, United States: Island Press.

Clark, J. R. (Ed.). (1995). Coastal zone management handbook. CRC Press.

Cobas, E. C. (2007). La Educación Ambiental en el uso de los recursos por las comunidades desde la perspectiva del Manejo Integrado de Zonas Costeras. Santiago, (111), 101-114.

Contraloría General de la República (CGR). (2005). Memoria Anual (281 p.). San José, Costa Rica.

Correa, S., Turbay, S., \& Vélez, M. (2012). Conocimiento ecológico local sobre ecosistemas marinos en dos comunidades costeras: El Valle y Sapzurro. Revista Gestión y Ambiente, 15 (2): 17-32 p. 
Decreto Ejecutivo $\mathrm{N}^{\circ}$ 34433-MINAE. Reglamento de la Ley de Biodiversidad de abril de 2008.

Departamento Nacional de Planeación (DNP). 2008. Elementos básicos para el Manejo Integrado de Zonas Costeras. DNP. Colombia

Honey, M., Vargas, E. \& Durham W. 2010. Impacto del turismo relacionado con el desarrollo en la costa Pacífica de Costa Rica (Informe ejecutivo). Center for Responsible Travel. San José, Costa Rica.

Instituto Nacional de Estadística y Censos (Costa Rica), INEC. (2011). X Censo Nacional de Población y VI de Vivienda: Resultados Generales / Instituto Nacional de Estadística y Censos (INEC). 1 ed. San José, C.R.

Lagarde, M. (1997). Claves feministas para el poderio y autonomía de las mujeres. Memoria (1 ed.). Managua, Nicaragua: Puntos de Encuentro.

Lemary, M., Vaughan, W., Rodríguez, D., Christy, F., Agüero, M., \& Rodríguez, J. (1997). Coastal and Marine Resources Management. Inter-American Development Bank. Draft. 50p.

Ley Orgánica del Ambiente, Ley N7554 del 4 de octubre de 1995.

Marshall, N.A., Marshall, P.A., Abdulla, A., \& Rouphael, T. (2010). The links between resource dependency and attitude of commercial fishers to coral reef conservation in the Red Sea. Ambio, 39:305-313.

Morales-Ramírez, A., Silva-Benavides, M., \& GonzálezGairaud, C. (2010). La gestión integrada de la zona costera en Costa Rica: experiencias y perspectivas. En: J.M., Barragán-Muñoz (Ed). Manejo costero integrado y política pública en Iberoamérica. Un diagnóstico. Necesidad de cambio (pp. 41-70). Cádiz, España: Red IBERMAR (CYTED).

Morales-Ramírez, A. (2013). Un análisis sobre la situación de algunos de nuestros ecosistemas costeros: necesidad de una gestión integrada. Ambientico, 230-231: $16-26$.

Naranjo, H. (2010). Caracterización de los sistemas operacionales, modalidades y artes de pesca utilizados para la captura de la langosta Panulirus gracilis (Streets, 1871) en Guanacaste, Costa Rica. Revista Ciencias Marinas y Costeras, 2: 73-82.

Pereira, J \& Sierra, L. (2011). Estrategia de manejo de los recursos marinos y costeros en Isla Uvita, Limón, Costa Rica. Revista Ciencias Marinas y Costeras, 1: $127-143$

Pernudi, V., Solórzano, N., Sandoval, I., \& Vargas, R. (2004). Percepciones de la ciudadanía costarricense sobre el ambiente en el país. Pulso Nacional, Universidad Nacional, 33:19.
Pernudi, V., Solórzano, N., Sandoval, I., \& Vargas, R. (2005). Percepciones de la ciudadanía costarricense sobre el ambiente en el país. Pulso Nacional, Universidad Nacional, 39:113.

Quesada, M. (2006). Participation and fisheries management in Costa Rica: from theory to practice. Marine Policy, 30:641-650.

Rowe, C. (2011). Fishing Away Marine Conservation: Poverty, Resource Dependence, and Poor Management in Cuajiniquil, Costa Rica. Tesis para optar por el grado de bachiller en Artes, Amherst College, Departamento de Estudios Ambientales. 97 p.

Sampieri, R. H., Collado, C. F., Lucio, P. B., \& Pérez, M. D. L. L. C. (1998). Metodología de la investigación. México: McGraw-Hill.

Solís-Rivera, V., Fonseca-Borrás, M. \& Barguil-Gallardo, D. (2012). Conservación marina en el Parque Nacional Santa Rosa. Las comunidades de Cuajiniquil, La Cruz y El Jobo. En D. Barguil-Gallardo, V. SolísRivera \& M. Fonseca-Borrás (Eds.). Monografia. Estudio regional sobre las dimensiones sociales en el Manejo de Áreas Marinas Protegidas: Casos Costa Rica, Nicaragua, Honduras y Panamá (p. 163). India: International Collectives in Support of Fishworkers (ICSF).

Soto, M. (2014, Feb 15). Pescadores artesanales luchan por primera zona de pesca sostenible. La Nación, p. 4.

Westmacott, S. (2002). Where should the focus be in tropical integrated coastal management? Coastal Management, 30: 67-84.

Worm, B., Barbier, E., Beaumont, N., Duffy, J., Folke, C., Halpern, B., Jackson, J.,...Watson, R. (2006). Impacts of biodiversity loss on ocean ecosystem services. Science, 314: 787-790.

\section{Referencias de Internet}

INCOPESCA. 2013. Estadísticas Pesqueras de Guanacaste 1992-2009. (Consultado 13 agosto 2013), http:// www.incopesca.go.cr).

Marine Stewardship Council (MSC). 2012. MSC Certification Requirements (Consultado el 24 de marzo de 2014, http://www.msc.org/documentos/estandares$\mathrm{msc} /$ Principios-y-Criterios-para-la-Pesca-Sustentable.pdf

Poder Judicial. 2013. Comunicado de Prensa. SC-CP-3013. 7 agosto de 2013. (Consultado 19 agosto 2013 Sala constitucional prohíbe el uso de redes de arrastre para la pesca de camarón, http://www.poder-judicial. go.cr). 
